\title{
Soft sub-leading divergences in Yang-Mills amplitudes
}

\section{Eduardo Casali}

Department of Applied Mathematics and Theoretical Physics, Wilberforce Road, Cambridge CBЗ OWA, U.K.

E-mail: e.casali@damtp.cam.ac.uk

ABSTRACT: In this short note I show that the soft limit for colour-ordered tree-level YangMills amplitudes contains a sub-leading divergent term. This term is analogous to those found recently by Cachazo and Strominger for tree-level gravity amplitudes.

KEYwords: Scattering Amplitudes, Gauge Symmetry

ARXiv EPRINT: 1404.5551 


\section{Introduction}

Recently, soft limits have been investigated from the point of view of the asymptotic symmetries of flat spacetime [1-3]. In particular, Weinberg's soft graviton theorem has been derived from the action of the supertranslations of the BMS group on the gravitational S-matrix. The BMS group can be extended by considering transformations that act singularly on the sphere at infinity $[4,5]$ These new transformations, called superrotations, act on the S-matrix. This action has been conjectured to be connected to a new universal subleading divergence around the soft limit of gravitational scattering amplitudes [6]. With that in mind, I derive the analogous sub-leading divergence of colour-ordered Yang-Mills amplitudes and show that they are given by an angular momentum generator acting on the scattering amplitude. It is not unreasonable to conjecture that this sub-leading term might be connected to symmetries of the asymptotic data of Yang-Mills on flat spacetime. Sub-leading effects in gauge theory amplitudes were first studied in [7] and the results extended to more general scattering states in $[8,9]$. In the context of gravity they were first studied in [10]. These sub-leading terms also have a strong resemblance to structures found in the systematic expansions of amplitudes carried out in [11-13].

The usual soft limit is taken by introducing a small parameter $\epsilon$ which rescales one of the gluons as

$$
\lambda_{s} \rightarrow \sqrt{\epsilon} \lambda_{s}, \quad \tilde{\lambda}_{s} \rightarrow \sqrt{\epsilon} \tilde{\lambda}_{s} .
$$

However, in order to see the sub-leading term it is better to use a related limit. This limit is a holomorphic version of the soft-limit [14], and is related to the usual one by the little group transformation

$$
\mathcal{M}(\{\sqrt{\epsilon} \lambda, \sqrt{\epsilon} \tilde{\lambda},+1\})=\epsilon \mathcal{M}(\{\epsilon \lambda, \tilde{\lambda},+1\}) .
$$

Notice that this relation differs from the one for gravity by a power of $\epsilon$, so in Yang-Mills there is no sub-sub-leading divergence as in gravity. The claim is that gluon amplitudes behave under a holomorphic soft-limit as

$$
\mathcal{M}_{n+1}\left(\left\{\epsilon \lambda_{s}, \tilde{\lambda}_{s}\right\}, 1, \ldots, n\right)=\left(\frac{1}{\epsilon^{2}} S^{(0)}+\frac{1}{\epsilon} S^{(1)}\right) \mathcal{M}_{n}(1, \ldots, n)+\mathcal{O}\left(\epsilon^{0}\right) .
$$

Here $S^{(0)}$ is the usual soft limit for colour-ordered YM amplitudes, and the sub-leading term is

$$
S^{(1)}=\frac{E_{\nu} q_{\mu} J_{a}^{\mu \nu}}{q \cdot k_{a}}
$$

where the particle labelled by $a$ is adjacent to the particle going soft. Notice that this term is gauge-invariant due to the antisymmetry of $J^{\mu \nu}$, so it has more in common with what was called $S^{(2)}$ in [6]. In spinor notation this can be written as

$$
\frac{E_{\nu} q_{\mu} J_{a}^{\mu \nu}}{q \cdot k_{a}}=\frac{\mu_{\alpha} \tilde{\lambda}_{s \dot{\alpha}}}{\langle\mu s\rangle} \frac{\lambda_{s \beta} \tilde{\lambda}_{s \dot{\beta}}}{\langle s a\rangle[s a]}\left(\varepsilon^{\alpha \beta} \tilde{J}^{\dot{\alpha} \dot{\beta}}+\varepsilon^{\dot{\alpha} \dot{\beta}} J^{\alpha \beta}\right)
$$

where

$$
J^{\alpha \beta}=\lambda^{\alpha} \frac{\partial}{\partial \lambda_{\beta}}+\lambda^{\beta} \frac{\partial}{\partial \lambda_{\alpha}}, \quad \tilde{J}^{\dot{\alpha} \dot{\beta}}=\tilde{\lambda}^{\dot{\alpha}} \frac{\partial}{\partial \tilde{\lambda}_{\dot{\beta}}}+\tilde{\lambda}^{\dot{\beta}} \frac{\partial}{\partial \tilde{\lambda}_{\dot{\alpha}}} .
$$




\section{Proof}

The proof follows closely the one given in [6], so I adopt their notation in what follows. First, consider the colour-ordered amplitude for the scattering of $n+1$ particles in Yang-Mills at tree-level, $\mathcal{M}_{n+1}(s, 1, \ldots, n-1, n)$. I denote by $s$ the soft particle which, for simplicity, I'll assume to have helicity $h_{s}=+1$. The amplitude without the momentum conserving delta function will be called $M$. Next, deform this stripped amplitude using the BCFW shift

$$
\lambda_{s}(z)=\lambda_{s}+z \lambda_{n}, \quad \tilde{\lambda}_{n}(z)=\tilde{\lambda}_{n}-z \tilde{\lambda}_{s} .
$$

The amplitude factorizes as

$$
M_{n+1}=\sum M_{L}\left(s\left(z^{*}\right), 1, \ldots, j, I\right) \frac{1}{P_{I}^{2}} M_{R}\left(-I, j+1, \ldots, n\left(z^{*}\right)\right)
$$

where the sum is over the set of ordered particles as well as the helicities of the internal particle.

In the soft limit the only interesting term in the sum is the one where $j=1$. In this case, $M_{L}$ is a three particle amplitude. The other terms are finite in the soft limit. The proof is the same as the one given in appendix A of [6]. Therefore in the following I'll drop all other terms except

$$
M_{L}\left(s\left(z^{*}\right), 1, I\right) \frac{1}{P_{I}^{2}} M_{n}\left(-I, 2, \ldots, n-1, n\left(z^{*}\right)\right) .
$$

In this term the pole is located at

$$
\left(k_{s}\left(z^{*}\right)+k_{1}\right)^{2}=0, \quad z^{*}=-\frac{\langle 1 s\rangle}{[1 n]}
$$

which fixes the internal spinor to be

$$
\lambda_{I}=\lambda_{1}, \quad \tilde{\lambda}_{I}=\frac{\langle n s\rangle}{\langle n 1\rangle} \tilde{\lambda}_{s}+\tilde{\lambda}_{1}
$$

The three point amplitude is non-zero when $h_{1}=-h_{I}$ and both choices of helicity of the internal particle give the same contribution. Combining terms, the BCFW recursion gives

$$
M_{n+1}(s, 1, \ldots, n)=\frac{\langle n 1\rangle}{\langle n s\rangle\langle s 1\rangle} M_{n}\left(-I, 2, \ldots, n\left(z^{*}\right)\right)+\ldots
$$

Now rescaling $\lambda_{s} \rightarrow \epsilon \lambda_{s}$, the above equation becomes

$$
\begin{aligned}
& M_{n+1}\left(\left\{\epsilon \lambda_{s}, \tilde{\lambda}\right\},\left\{\lambda_{1}, \tilde{\lambda}_{1}\right\}, \ldots,\left\{\lambda_{n}, \tilde{\lambda}_{n}\right\}\right) \\
& \left.\quad=\frac{1}{\epsilon^{2}} \frac{\langle n 1\rangle}{\langle n s\rangle\langle s 1\rangle} M_{n}\left(\left\{\lambda_{1}, \tilde{\lambda}_{1}+\epsilon \frac{\langle n s\rangle}{\langle n 1\rangle} \tilde{\lambda}_{s}\right\}, \lambda_{2}, \tilde{\lambda}_{2}\right\}, \ldots,\left\{\lambda_{n}, \tilde{\lambda}_{n}+\epsilon \frac{\langle s 1\rangle}{\langle n 1\rangle} \tilde{\lambda}_{s}\right\}\right) .
\end{aligned}
$$

$M_{n}$ is finite when $\epsilon \rightarrow 0$ and corresponds to the stripped $n$ point amplitude. Restoring the momentum conserving delta functions to the amplitude and expanding it to first order around $\epsilon=0$ gives

$$
\begin{array}{r}
\left.\mathcal{M}_{n}\left(\left\{\lambda_{1}, \tilde{\lambda}_{1}+\epsilon \frac{\langle n s\rangle}{\langle n 1\rangle} \tilde{\lambda}_{s}\right\}, \lambda_{2}, \tilde{\lambda}_{2}\right\}, \ldots,\left\{\lambda_{n}, \tilde{\lambda}_{n}+\epsilon \frac{\langle s 1\rangle}{\langle n 1\rangle} \tilde{\lambda}_{s}\right\}\right) \\
=\left(1+\epsilon \frac{\langle n s\rangle}{\langle n 1\rangle} \tilde{\lambda}_{s} \cdot \frac{\partial}{\partial \tilde{\lambda}_{1}}+\epsilon \frac{\langle 1 s\rangle}{\langle 1 n\rangle} \tilde{\lambda}_{s} \cdot \frac{\partial}{\partial \tilde{\lambda}_{n}}\right) \mathcal{M}_{n}(\epsilon=0) .
\end{array}
$$


After multiplying by the soft factor, the expression for the amplitude is

$$
\begin{aligned}
& \mathcal{M}_{n+1}\left(\left\{\epsilon \lambda_{s}, \tilde{\lambda}_{s}\right\}, 1, \ldots, n\right)=\left(\frac{1}{\epsilon^{2}} S^{(0)}+\frac{1}{\epsilon} S^{(1)}\right) \mathcal{M}_{n}(1, \ldots, n)+\mathcal{O}\left(\epsilon^{0}\right) \\
& S^{(0)}=\frac{\langle n 1\rangle}{\langle n s\rangle\langle s 1\rangle}, \quad S^{(1)}=\frac{1}{\langle s 1\rangle} \tilde{\lambda}_{s} \cdot \frac{\partial}{\partial \tilde{\lambda}_{1}}+\frac{1}{\langle n s\rangle} \tilde{\lambda}_{s} \cdot \frac{\partial}{\partial \tilde{\lambda}_{n}}
\end{aligned}
$$

as claimed.

Notice that there is no sub-sub-leading divergence in this limit as is the case for gravity. Here the term at this order is finite and mixes with the rest of the BCFW recursion terms. Another important difference is that while $S^{(0)}$ is universal for tree-level amplitudes, at loop-level this soft factor receives corrections $[15,16]$, while the gravitational soft-factor does not. It is expected that the sub-leading divergence $S^{(1)}$ will also receive quantum corrections.

Nevertheless, this tree-level data is still interesting. The sub-leading divergences are proportional to the angular momentum operator just like in gravity, and might be derived by asymptotic methods as was done for the usual soft limit in [1]. On the other hand, this sub-leading divergence is gauge-invariant by itself, not requiring conservation of linear or angular momentum much like the sub-sub-leading divergence of the soft limit in gravity. It would be interesting to investigate the extent to which these terms are in any sense universal or connected to properties of the asymptotic boundary of flat spacetimes.

\section{Acknowledgments}

I'm grateful to D. Skinner and T. Adamo for discussions, to L. Greenspan for comments on the first draft, and to D. Skinner for suggesting this problem. This work is supported by the Cambridge Commonwealth, European and International Trust.

Open Access. This article is distributed under the terms of the Creative Commons Attribution License (CC-BY 4.0), which permits any use, distribution and reproduction in any medium, provided the original author(s) and source are credited.

\section{References}

[1] A. Strominger, Asymptotic symmetries of Yang-Mills theory, JHEP 07 (2014) 151 [arXiv: 1308.0589] [INSPIRE].

[2] A. Strominger, On BMS invariance of gravitational scattering, JHEP 07 (2014) 152 [arXiv: 1312.2229] [INSPIRE].

[3] T. He, V. Lysov, P. Mitra and A. Strominger, BMS supertranslations and Weinberg's soft graviton theorem, arXiv:1401.7026 [INSPIRE].

[4] G. Barnich and C. Troessaert, Supertranslations call for superrotations, PoS (CNCFG2010) 010 [arXiv: 1102.4632] [INSPIRE].

[5] G. Barnich and C. Troessaert, BMS charge algebra, JHEP 12 (2011) 105 [arXiv:1106. 0213] [INSPIRE]. 
[6] F. Cachazo and A. Strominger, Evidence for a new soft graviton theorem, arXiv:1404.4091 [INSPIRE].

[7] F.E. Low, Bremsstrahlung of very low-energy quanta in elementary particle collisions, Phys. Rev. 110 (1958) 974 [inSPIRE].

[8] T.H. Burnett and N.M. Kroll, Extension of the low soft photon theorem, Phys. Rev. Lett. 20 (1968) 86 [inSPIRE].

[9] V. Del Duca, High-energy bremsstrahlung theorems for soft photons, Nucl. Phys. B 345 (1990) 369 [INSPIRE].

[10] D.J. Gross and R. Jackiw, Low-energy theorem for graviton scattering, Phys. Rev. 166 (1968) 1287 [INSPIRE].

[11] C.D. White, Factorization properties of soft graviton amplitudes, JHEP 05 (2011) 060 [arXiv:1103.2981] [INSPIRE].

[12] E. Laenen, G. Stavenga and C.D. White, Path integral approach to eikonal and next-to-eikonal exponentiation, JHEP 03 (2009) 054 [arXiv:0811.2067] [INSPIRE].

[13] E. Laenen, L. Magnea, G. Stavenga and C.D. White, Next-to-eikonal corrections to soft gluon radiation: a diagrammatic approach, JHEP 01 (2011) 141 [arXiv:1010.1860] [INSPIRE].

[14] N. Arkani-Hamed, F. Cachazo and J. Kaplan, What is the simplest quantum field theory?, JHEP 09 (2010) 016 [arXiv:0808.1446] [INSPIRE].

[15] L.J. Dixon, L. Magnea and G.F. Sterman, Universal structure of subleading infrared poles in gauge theory amplitudes, JHEP 08 (2008) 022 [arXiv:0805.3515] [INSPIRE].

[16] L.J. Dixon, E. Gardi and L. Magnea, On soft singularities at three loops and beyond, JHEP 02 (2010) 081 [arXiv:0910.3653] [INSPIRE]. 